\title{
N.J. Fisch
}

MARCH 1985

\section{MSTER}

\section{PLASMA PHYSICS \\ LABORATORY}

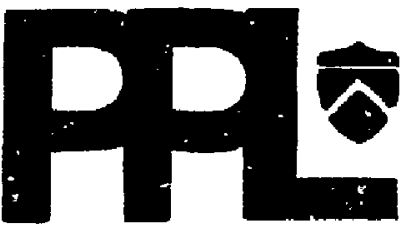

PRINCETON UNIVERSITY

PRINCETON, NEW JERSEY

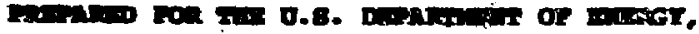

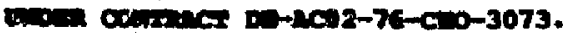




\author{
N. J. Fisch \\ Plasma Physics Laboratory, Princeton Univergity \\ P. O. Box 451 \\ Princeton, New Jersey 08544
}

\begin{abstract}
A plasma in contact with an external source of power, especially a source that interacts specifically with high-velocity electrons, exhibits transport properties, wuch as conductivity, different from those of an isolated plasma near thermal equilibrium. This is true even when the bulk of the particles in the driven plasma are near thexmal equilibrium. To describe the driven plasma we derive an adjoint equation to the inhomugeneous, linearized, dynamic Boltzmann equation. The Green's functiong for a variety of plasma responses can then be generated. It is possinle to modify the Chapman-Enskog expansion in order to incorporate the response functions derived here.
\end{abstract}

\title{
DISCLAIMER
}

This repart wa preparai as an cocount of wort sponeored by an aseacy of the Unilod State.

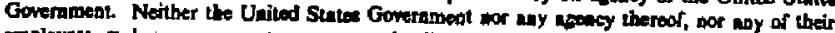

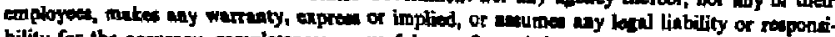
bility for the eccuracy, conplocercem, or ubefulnew of any information, apparafus product, or

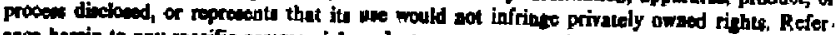

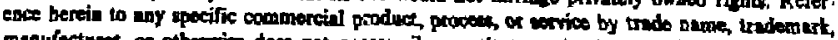

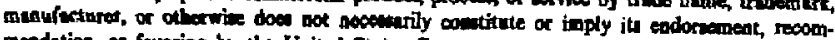

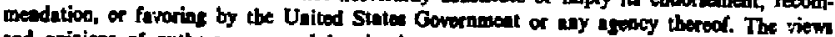

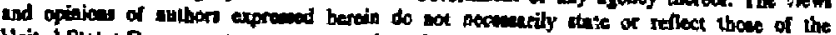
Unitod Stato Goveraneat or any wency thereol. 


\section{T. IINTRODUCTION}

The expansion developed by Chapman and Enskog ${ }^{1}$ provides a generally useful description of transport in a plasma. This expansion, which applies when the mean-free path of particles is small compared to other lengths of interest, has been the starting point for describing plasmas near thermal equilibrium. At the heart of this method is a reduced description of each plagma species in terms of macroscopic fluid variables, namely, the dengity, temperature, and drift. It is these properties of the plasma which are transported in configuration space in order to describe the evolving plasma. The transport numbers, such as the conductivity or the coefficienta governing particle and heat diffusion, are calculated by considaring smal! perturbations from thermal equilibrium. It is well-known, however, that certain phenomena are not described well by this method, namely, those processes involving the hiqhly energetic particles, particularly electrons, which collide but insrequently. For example, the plasma conductivity, the sscalled spitzer-Harm conductivity, ${ }^{2}$ is gtrictly derived only in the limit of the de electric field, E, vanishing. For E finite, important corrections occur due to the high-velocity electrons, which tend to run away. If many of. these electrons run away, the Spitzer-harm conductivity may no Iriger provide a useful description. The regime in which snly few electrons run away, where conductivity is a good description, is the small-mean-free-path limit, which arises for E small enough.

In the case of driven plasmas, however, the spitzer-Härm conductivity may not be a useful approximation even for $E \rightarrow 0$. These plasmas are not near thermal equilibrium; they are actively heated by an external source of radiation or particles. One example of a driven plasma that is not described well by the chapman-Enskog expansion, and hence not by spitzer-Harm 
conductivity, is a plasma in contact with external radiation that interacts specifically with the fast, collisionless electrons. This is a situation chat commonly arises in rf-heated plasmas, particularly when the purpose is to generate toroidal current. The deviation from spitzer-Harm conductivity is aue to the larger number of fast electrons. These electrons have long meanfree paths, and even though small in number compared to the bulk electrons, they may play a large part in carrying current. ${ }^{3}$

In this report, we develop a description suitable for driven plasma. we consider those plasmas for which it is valid to apply the fundamental chapmanEnskoq approximation, that collisions are large enough to create a nearly Maxwellian distribution of particle velocities. We use this approximation to linearize the Boltzmann collision operator, and as a result we obtain the dynamic linearized, inhomogeneous Boltzmann equation. plasma responseg to arbitrary wave excitation affecting the fast electrong are then determined by solving an adjoint equation. The result is an improved characterization of the relatively collisioniess phenomena associated with the external excitation of the plasma. A modification of the Chapman-Enskog expansion then allows a dual characterization of driven plasnas; a slowly evolving fluid description of the bulk properties and a aynamic response, linear in the excitation, for the triven relatively collisionless processes.

In order to put the present work in perspective, let us briefly review some of the approaches taken to this problem. Response functions for the current generated per power dissipated, $J /{ }{ }_{d}$, were central to our early understanding of both beam-driven currents, 4 and wave-driven currents with low and high ${ }^{6}$ phase velacity waves. The early work, however, was characterized by a crude one-dimensional model of velocity space, and was unable to predict, for example, current drive by electron cyclotion (ECRH) 
waves. Figch and Boozer ${ }^{7}$ used glowing-down equations to formulate the problem in two dimensions in velocity space. This gave the ficst precise response function for the current-drive efficiency and predicted the BCRH current-drive effect.

An improved derivation of the 2-D Fisch-Boozer response furlction was provided by Antonsen and Chu. ${ }^{B}$ Drawing upon neoclagsical techniques, these authors extended the Fisch-Boozer efficiency calculation to toroidal geometry by writing an adjoint equation to the steady-gtate linearized Boltzmann equation. Hirstman ${ }^{9}$ and Taguchi ${ }^{10,11}$ employed a similar technigue to solve similar equations. Using the adjoint equation, Antonsen and Yoshioka ${ }^{12}$ have calculated rf-induced particle transport across field lines. The Antonsen and Chu approach was also generalized to include a small dc electric field in order to evaluate the conductivity of a ditiven plasma. 3

In order to interpret the recent high efficiency pur ramp-up experiments, ${ }^{13}$ response functions had to be identified and evaluated in the presence of a strong dc electric field. The most straightforward approach, adspted by Fisch and Karney, ${ }^{14}$ qeneralized the Fisch-Boozer slowing-down equations to include the strong field. In addition to the current-drive efficiency, this work also identified the Green's function for runaway production. A comparison by karney et al.$^{15}$ of this theory and the experiment showed very good agreement.

A better approach to the strong-field case is to build upon the AntonsenChu work. The present work seeks to to this. Here, ari adjoint equation is written for the dynamic, Iinearized Boltzmann equation, allowing for the presence of a strong dc electicic field. Also, in addition to a response function for the current, other response functions are identified and evaluater. It is interesting to note that were this adjoint equation solved 
in the high-velocity limit by the method of chacacteristics, the characteristic equations would be the Fisch-Boozer slowing-down equations.

The adjoint formalism developed here also draws heavily on insights gathered in solutions to the homogereoug Boltzmann equation, $16-18$ which exploit, too, the self-adjoint property of the collision integral. Here, however, the focus is on the effects associated with the wave-induced large deviations from Maxwellian velocity distributions.

The paper is organized as follows. In sec. II we discuss the validity and the utility of the linearization of the Boltzmann equation. In sec. III we drrive the adjoint equation and we write the general Green's function response. This is our principal result. In Sec. IV we give severai exampleg of the utiliry of this general equation. A morified Chapman-Enskog expansion, that includes the derived response functions in addition to the normal fluid terms, is qiven in se:. V. In sec. VI, we discuss ways of implementing numerical tcols, based on these results, suitable for modeling a plasma experiment. A sumary of our findings is given in sea. VII.

\section{LINEARIZ ED BOLTZMANA EQUATION}

The Boltzmann equation provides an excellent description of the evolving plasma, but it is too complicated to apply directly to most problems of interegt. Certain approximations, however, allow us to extract information from this equation reliably and efficiently. The most helpful simplifications that are unique to the wave-driven plasma cely on the localization in velocity space of the ware-driven fluxes and the fact that these fluxes often involve only very fast electrons.

To be specific, let us consider the electron distribution function $f(r, \vec{v}, t)$ driven by some wave-induced flux $\vec{\Gamma}(x, \vec{v}, t)$, evolving as described by the inhomogeneous Boltzman equation 


$$
\frac{d f}{d t}=c(f, f)+c\left(f_{r} f_{i}\right)-\frac{\partial}{\partial v} \cdot t
$$

where $C(f, f)$ repregents the self-collisions of electrons, $C\left(f_{,} f_{i}\right)$ repregents the scattering of electrons off ion distribution $f_{i}$, and the total time derivative, $d / d t$, is defined by

$$
\frac{a}{d t} \equiv \frac{\partial}{\partial t}+\vec{v} \cdot \frac{\partial}{\partial \vec{r}}+\vec{F} \cdot \frac{\partial}{\partial \vec{v}}
$$

where $\vec{F}$ is the acceleration due to the background dc electric field $\vec{E}$ and magnetic field $B$, i.e.,

$$
F=\frac{e}{m}(\vec{E}+\vec{v} \times \vec{B})
$$

To be sure, we generally expect that the magnetic field will be so large that the conventional expansicns learing, for example, to the drift kinetic equation are appropriate. To sclve or. (1), we will make use of any of these expansions, which are useful whether or not the electrons are subject to waveinduced fluxes. However, here, we shall focus only on approximations that are specific to the problem at hand.

The main effect of the injection of intense $r$-waves at high phase velocities parallel to the magnetic field $\vec{B}$ is to distort the electron distribution in the resonant region of velocity space. Waves at frequency u ara injecter with a range of parallei wave numbers $k_{\|}$such that $v_{1}<\omega / k_{\mu}<$ $v_{2}$. The region of the electron velocity space resonant with the waves is $v_{1}<$ $v_{\|}<v_{2}$, where $v_{\|}$is the electron velocity parallel to $\vec{B}$. In the resonait region the waves act to diffuse electrons, the so-called "quasilinear 

with electron eyclotron waves, will be nearly in the perpendicular direction. If the spectrum of waves is narrow in kpppace, then only electrons within a narrow band in Y p-space will be resonant, such as those electrons with $v_{I} \cong v_{\text {reg }}$. If this region is narrow and $v_{\text {res }} \gg v_{\text {Te' }}$, where $v_{\text {Te }}$ is the electron thermal velocity, then even if the electron distribution is distorted by the waves, the wave-induced flux $\vec{\Gamma}$ is of known direction and finite only for $v_{H}=v_{\text {res }}, v_{\perp}=v_{\mathrm{Te}}$, or in other words, $\vec{v} \simeq v_{\text {Ies }} \hat{i}_{\|}$. only the magnitude of $\vec{\Gamma}$, which for Landau damping depends on $\partial f / \partial v_{\|}$in the resonant region, remains unknown.

Knowledge of all but the magnitude of $\vec{\Gamma}$, tagether with the license to linearize Eq. (1) allows us to solve easily an important class of problems. Suppose that a certain power $\mathrm{P}_{\mathrm{g}}$ is absorbed from the waves by the resonant electrons. As a result, there may appear in the plasma a wave-induced current J. The quantity $J / \mathbb{P}_{d}$, which is a measure of the efficiercy of the current drive, is independent of the magnitude of $\vec{\Gamma}$, since both $J$ and $P_{d}$ are proportional to it. The ratio, however, does depend upon both the rection and velocity locale of $\vec{\Gamma}$, which could be inferred approximately, as ontlined above, without knowledge of the magnitude of $\vec{\Gamma}$. Other responses, such as the incremental bremmstrahlung radiation per power absorbed, can be conputed in the same manner. The quantity $J / P_{d}$, and other quantities that are similarly inferred, degcribe the response of the plasma to the absorption of wave energy by resonant electrons, and provide insight as to how to inject tinese waves in order to maximize a desired response.

The magnitude of $\overrightarrow{I^{\prime}}$ is also an important quantity, since all plasma responges will be proportional to it. This quantity, however, may be calculated by more crude means. Further discussion of this point is deferred to Sec. VI. 
III. ADJOIRT EOUATION

The linearization of the Boltzmann equation enables us to find responses to arbitrary wave-induced fluxes. Instead of solving the Boltzmann equation, we solve an adjoint equation for the Green's function. In this section, we derive the adjoint equation. For simplicity, we shall restrict our attention to the homogeneous case, $\partial / \partial \vec{x}=0$. However, we shall solve the problem in a finite velocity Jomain, which facilitateg a jumerical implementation.

There is a certain amount of arbitrariness in imposing a specific linearization. We shall adopt a linearization that will enable us to generalize easily the Chapman-Enskog expansion. We utilize the Eact that the heating of the bulk of $f$ occurs on a time scale long compared to the time scales for other processes of interest, while collisions, predominant in the bulk of $f$, assure for thermal velocities a nearly Maxwellian distribution. Thus, we linearize

$$
E=E_{m}(1+\phi)
$$

where

$$
f_{m} \equiv n\left(\frac{2 \pi T}{m}\right)^{-3 / 2} \exp \left(\frac{-\varepsilon}{T}\right)
$$

where density $\mathbf{n}$ and temperature $\mathbf{T}$ may be slow funotiong of time land would be of space, too, in a more general consideration of the problem), and we defined the kinetic energy $E \equiv m v^{2} / 2$. For simpljcity of presentation, we have made one further simplification in the chapman-Enskog approach by linearizing about a stationary rather than the more general drifting Maxwellian distribution. 
To find a unique solution with this procedure necessitates two compatibility conditions; we choose $n$ and $T$ such that $f_{m}$ (integrated over velocity space) contains the yame number of eledtrong and the sane amount of energy as doeg f. Accordingly, $\phi(\vec{v}, \vec{r}, t)$ is orthogonal both to $f_{m}$ and to $\varepsilon f_{m}$, namely

$$
\int_{v} E \phi d^{3} v=\int_{v} \varepsilon_{m} \phi d^{3} v=0
$$

where $V$ is the velocity space domain under consideration, which necessarily is finite in a numerical implementation of the method.

substituting Fq. (5) into Eq. (1), and specialiaing to the homogeneors case, $\partial / \partial_{r}^{+}=0$, we obtain

$$
L \phi \equiv \frac{\partial}{\partial t} f_{m} \phi+\vec{F} \cdot \frac{\partial}{\partial v_{v}^{+}} f_{m} \phi-c(\phi)=-\frac{\partial}{\partial \vec{v}} \cdot \vec{F}-\frac{\partial}{\partial t} f_{m}-\vec{F} \cdot \frac{\partial}{\partial \vec{v}} f_{m} \cdot
$$

where $\phi$ obeys a homogeneous initial condition and where we use

$$
\frac{\partial f_{m}}{\partial t}=\frac{\partial n}{\partial t} \frac{\partial \dot{f}_{m}}{\partial n}+\frac{\partial T}{\partial t} \frac{\partial f}{\partial T} \equiv \frac{\dot{n}}{n} f_{m}+\left(\frac{\varepsilon}{T}-\frac{3}{2}\right) \frac{\dot{T}}{T} f_{m}
$$

although $t_{m}$ is assumed to evolve on a time scale lerger than the scale $t$ on which $\phi$ evolves. In Eq. (9) we also adopted the convenjent notation

$$
c(\phi) \equiv c\left(f_{m}, f_{m} \phi\right)+c\left(f_{m} \phi, f_{m}\right)+c\left(f_{m} \phi, f_{i}\right)
$$

In order to assure the $\mathrm{Eq}$. (7) continues to be obeyed as $\dot{\phi}$ evolves 1 , time two solubility, or compatibility, conditions are applied to Fq. (g). To assure the orthogonality of $\phi$ to $f_{m}$, we integrate $8 q .(8)$ over $v$ to obtain 


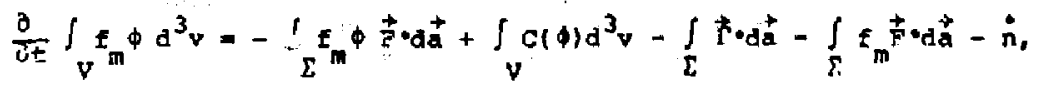

where $\Sigma$ is the surface in relocity space, bounding $v$, and $\dot{n}$ is shosen so that the right-hand sice of $\mathrm{Bq}$. (11) vanishes, In practice, we wish to choose $v$ large enough such that thi boundary terms to not introduce severe particle lasses, and such that particles wich do ieave through the boundary can be accounted for in some other simple way (such as free-sireaming), The physical implization of decreasung $\mathrm{n}$ as a result of runaway prcduction is that the hulk population is somewhat depleted of electrons and, hence, serves somewhat less effectively is a scatterer. Very fast electrong, i.e., thoge outgide the domain $v$, are inefective in contributing to the collisions of othor electrons. It is often sufficient to account for these electrons merely by

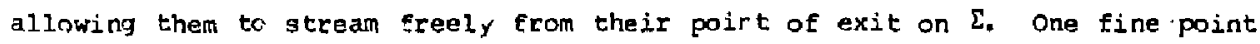
here is that we assume that the Maxwellian background is not confined to the bounded domain. This is consigtent with the other approximations made so far, and it is a convenience.

The second compatibility condition gives us $\dot{T}$, the temperature evolution of the background electrons, Multiplying Eq. (8) by $\mathrm{E}$, integrating over the domain V, and applying Eq. (7), we ger.

$$
\left(\frac{3}{2}\right)_{n \dot{T}}=-j \varepsilon\left[\frac{\partial}{\partial \vec{v}} \cdot \vec{\Gamma}+\vec{F} \cdot \frac{\partial}{\partial v} f_{m}+\vec{F} \cdot \frac{\partial}{\partial \vec{v}} f_{m} \phi-c(\phi)\right] d^{3} v
$$

where the first right-hand-side term above represents the energy input of the wave-driven flux, the second term vanishes, the third term contains the Joule heating effect of the rf-driven current, and the last term tends to zers as $v$ $+\infty$. 
Equation (B), together with Bq. (9) and the expression for $\dot{n}$ and $\dot{r}$, comprise an inhomogeneous, linear, integrondifeorential equation for $\$$. This equation, difficult to treat analytically, may be solved numerically. The evolving golution, $\phi(t)$, autonatically obeys the orthogonality conditions, so that the Iinearization, (5), continues to hold, which allowg the collision inteqral to retain its form. Rather than solve Dq.(8) for each excitation $\$$, however, we congider as adjoint equation instead.

To find an operator adjoint to $I$, we first define a commutative operation on the two functions $\phi\left(\vec{v}^{*}, t^{\prime}\right)$ and $\phi\left(\vec{v}, t^{\prime}\right)$ by

$$
[\phi, \psi]_{t} \equiv \int_{v} d^{3} v \int_{0}^{t} \phi(\vec{v}, t-\tau) \psi(\vec{v}, \tau) d \tau,
$$

where the operation [ ] is parameterized by $t$. Define a linear operator $D_{t}$ operating on $\psi\left(\vec{v}, t^{\prime}\right)$ by

$$
D_{t} \psi\left(\vec{v}, t^{\prime}\right) \equiv\left[f_{m} \frac{\partial}{\partial t^{\prime}} \Psi-f_{m} \vec{F}\left(\vec{v}, t-t^{\prime}\right) \cdot \frac{\partial}{\partial \vec{v}} \psi-c(\psi)\right]
$$

For functions $\phi$ and $\psi$ with homogeneous initial and boundary conditions, it may be shown that the operator $D_{t}$ is adjoint to the operator $I$ with respect to the inner product [ $]_{t}$, i.e.r

$$
\left[\phi, o_{t} \psi\right]_{t}=[\psi, \mathrm{L}, \phi]_{t}
$$

Suppose that $\phi\left(\vec{v}, t^{\prime}\right)$ and $\psi^{\prime}\left(\vec{v}, t^{\prime}\right)$ are restricted to functions orthogonal both to $f_{m}$ and to $E f_{m}$ over the domain $v$. Suppoge further that $\psi\left(\vec{v}, t^{\prime}\right)$ obeys the evolution equation 


$$
D_{t} \psi\left(\vec{v}, t^{\prime}\right)=q_{1} f_{m}+q_{2} \varepsilon_{m}
$$

where the constants $q_{1}$ and $q_{2}$ are chosen to assure that the orthogonality contitions on $\psi$ are obeyed subsequently, given that they are obeyed initially. The quantities $q_{1}$ and $q_{2}$ are, independent of $\dot{v}$, but are linear functionals of $\psi$. We now supplement 2 . (16) with inhomogeneous initial and borndary conditions on $\psi$, namely

$$
\begin{aligned}
& \psi\left(\vec{v}, t^{\prime}=0\right)=\psi_{0}(\vec{v}), \\
& \vec{s}_{\psi}\left(\vec{v}, t^{\prime}\right)=\vec{s}_{B}\left(\vec{v}, t^{\prime}\right) \text { on } \Sigma,
\end{aligned}
$$

where $\mathrm{I}$ is the boundary in velocity space to domain $y_{\text {, and }} \overrightarrow{\mathrm{s}}_{\psi}$ is the flux in velogity space asspciated with the operator $D_{t}, i, e .$,

$$
\vec{s}_{\psi}(\psi) \equiv-f_{m} \vec{F}(\vec{v}, t-\tau) \phi(\vec{v}, \tau)+\vec{s}_{c}(\phi),
$$

where $\vec{S}_{c}(\psi)$ is related to $C(\psi)$ by

$$
c(\psi)=-\frac{\partial}{\partial \vec{v}} \cdot \vec{s}_{c}(\psi)
$$

For the collision operator $c$, we have the property

$$
\int_{v}[\phi c(\phi)-\psi c(\phi)] \mathrm{d}^{3} v=\int_{\Sigma}\left[\phi \vec{s}_{c}(\psi)-\psi \overrightarrow{s_{c}}(\phi)\right] \cdot d \vec{a}
$$

For $v \rightarrow \infty$, the surface terms vanish and we have the well-known self-adjoint property of the collision operator. For our present needs, however, we shall keep the boundary terms. 
We take the inner product of and $D_{t} \psi_{\text {, where }}$ obeys $D q$. (B) with homogeneous initial condition and $\psi$ obev̧s $\mathbb{Z} q 5 .(16)$ and (1\%). We need to specify a boundary condition on Bq. (B) too, for our present purposes let us assume we know $s_{c}(\phi)$ on the boundary [but, we shall, in Dq. (23), give a knore useful, less general, condition]. Exploiting the orthogonality properties of both $\phi$ and $\psi$, we obtain

$$
\begin{aligned}
\int_{v} d^{3} v & f_{m} \phi(\vec{v}, t) \psi_{0}(\vec{v})+\int_{0}^{t} d \tau \int_{\Sigma} \phi(\vec{v}, \tau) \vec{s}_{B}(\vec{v}, t-\tau) \cdot d \vec{a} \\
& =\int_{v} d^{3} v \int_{0}^{t} d \tau \vec{\Gamma}_{*}(\vec{v}, t-\tau) \cdot \frac{\partial}{\sigma_{v}^{t}} \psi(\vec{v}, \tau) \\
& +\int_{0}^{t} d \tau \int_{\Sigma} \psi(\vec{v}, t-\tau)\left[\vec{\Gamma}_{*}+\vec{s}_{c}(\phi)\right] \cdot d \vec{a}
\end{aligned}
$$

The driven flux

$$
\vec{\Gamma}_{*}(v, t) \equiv \vec{\Gamma}(v, t)+\vec{F}(v, t) E_{m}
$$

is the sum of the wave-induced $f . u x \vec{I}$ and the flux induced by the background fields that tend to accelerate the background Maxwellian distribution. The latter flux leads to the ohmic current.

Equation (21) is a principal result of this work. Both $\phi_{0}$ and $\vec{s}_{B}$ are arbitrary functions, and they may be used to express any moment of $\phi$ either in the interiof of $v$ or on its bounding surface $\Sigma$, Depending on the desired moment of $\phi$, a different Green's funotion, $\psi$, is solved for by Eq-i. (16) and (17). Once $\Psi$ is known, the right-hand side of Eq. (21) may be evaluated for arbitrary wave induced fluxes $\vec{\Gamma}$. 
Note that in generalizing the gteady+gtate adjoint equations to include tine dependence, several significant steps were taken. The inner product, with respect to which the adjoint property is defined, was changed to include a convolution over time. Also note that the orthogonality conditions are imposed here to assure the continued validity of linearization in Eq. (5), and of the resulting simplification of the collision operator. These conditions reduce to the compatibility conditions of the steady-gtate theory in the limit $\partial / \partial t+0$. However, while the impogition of these conditions in the dynanic case results in considerable mathematical simplification, it is not necessary here for the existence of solutiong.

\section{EXAMPLES}

In this section, we give several examples showing how Bq. (21) may be used to find interesting plasma responses. For example, in addition to the current, the rf-induced runaway rate may be computed. A second example shows how contributions to the rf-induced plasma current for for that matter radiation, etc.)' can be separated into runaway and noncunaway contributions. A third example shows how analytic progregs can be made, using Eq. (21), to find the rf-induced conductivity in certarn limiting cases.

To calculate how rf waves affect the ruraway production rate, we must first be precise in defining a runaway electron. If the parallel electric field points always in one direction (i.e., electrons are pushed unidirectionally), then electrons can be accelerated by this field to high enough energy that they overcome the dymanic frictional force of collisions. If the domain $v$ is large, then an electron accelerated from the bulk of the distribution to the boundary $\Sigma$ may be deemed a rumaway, for frictional torces are extremely unlikely to retum this electron to the domain $V$. It is 
convenient to separate the houndary $\Sigma$ into two parts; an electron appearing on $\Sigma_{\text {out }}$ leaveg the domain $v$, while an electron appearing on $\Sigma_{\text {in }}$ is forcer into the domain $v_{r}$ and $\Sigma$ is the union of $\Sigma_{\text {out }}$ and $\Sigma_{\text {in }}$. For large $v_{\text {, it is }}$ primarily the electric field that determines this dichotomy. A more precise separation of $\Sigma$ takes into account the small, but finite, inwardly (i.A., into v) pointing collisional force in adrition to the force of the electric field, so that $\Sigma_{\text {out }}$ is slightly smaller than $\Sigma_{\text {in }}$. All culaway electrons appear at: some time on $\Sigma_{\text {out }}{ }$ and all electrons that appear on $\Sigma_{\text {out }}$ are runaways, freely accelerating out of the domain $v$.

Equivalently, we can imagine solving a problem in which collisions operate within the domain $v$, but the collision operator vanishes outside this domain, allowing electrons to stream freely. Where the collision operatur vanishes. the governing equation reverts from parabolic to hyperbolic. It is then correct to impose a boundary condition on just $\Sigma_{i n}$, rather than on the Eull boundary $\varepsilon$. The adjoint equation, on the other hand, is well-posed when a houndary condition is imposed only on $\Sigma_{\text {out }}$.

Define a flux $\vec{s}_{\phi}(\phi) \equiv \phi \mathrm{f}_{\mathrm{m}} \overrightarrow{\mathrm{F}}+\vec{s}_{\mathrm{c}}(\phi)$. Then, on $\Sigma_{\text {in }}$, we specify $\vec{s}_{\phi}(\phi)$, the incoming flux into domain $v$. For the runaway problem, and most other problems of interest, this flux is zero. For the adjoint equation, we require that $\vec{s}_{c}(\phi)=0$ on $\Sigma_{\text {in }}$. on $\Sigma_{\text {out }}$, the opposite holds. Here we specify $\vec{s}_{c}(\phi)=0$, indicating that runaway electrong eventually stream freely, We are then left with the freedom to choose $\vec{s}_{\psi}(\phi)$ in order to pose the adjoint equation.

Recognizing the vanishing of $\vec{s}$ on $\Sigma_{i n}$ and the vanishing of $\vec{s}_{c}$ on $\Sigma_{\text {out' }}$ we can manipulate $\mathrm{Eq}$. (21) into the more useful form 


$$
\begin{aligned}
& \int_{v} d^{3} v f_{m} \phi(\vec{v}, t) \Psi_{0}(\vec{v})+\int_{0}^{t} d \tau \int_{\Sigma_{\text {out }}} \phi(v, \tau) \vec{s}_{B}(\vec{v}, t-\tau) \cdot d \vec{a} \\
& \quad=\int_{v} d^{3} v \int_{0}^{t} d \tau \vec{\Gamma}_{*}(\vec{v}, t-\tau) \cdot \frac{\partial}{\partial \vec{v}} \psi(\vec{v}, \tau)+\int_{0}^{t} d \tau \int_{\Sigma} \psi(v, t-\tau) \overrightarrow{F_{*}}(\vec{v}, \tau) \cdot d \vec{a} \cdot
\end{aligned}
$$

The above formulation also allows a slignt refinement. The collisional slowing down may be distinguished from the collisional induced diffusion. Requiring that only the collisional diffusion vanish at the houndary gives a more accurate calculation of both the total flux at the boundary and the demarcation between $\Sigma_{\text {in }}$ and $\Sigma_{\text {out }}$. For example, for the runaway problem, the flux at the boundary may be expressed as the sum $\hat{i}_{d} \in E_{d} / m-v_{s} \vec{v}_{,}$where $v_{g} \vec{v}$ represents the dynamical friction due to collisions. The boundary $\Sigma_{\text {out }}$ is then defined to exist where this sum points outward from the domain $v$.

Let us find now the response function for the $r f$-induced funaway rate. The number of $r f$-induced runaway electrons, $N_{R^{\prime}}$ appearing on $\sum_{\text {out }}$ between time $\tau=0$ and time $\tau=t$ may be written as

$$
\mathrm{N}_{\mathrm{R}}=\int_{0}^{t} \mathrm{a} i \int_{\text {out }} \overrightarrow{\mathrm{s}} \cdot \overrightarrow{\mathrm{a}} \overrightarrow{\mathrm{a}}
$$

where $\vec{s}=\left(\hat{i}_{\|} \in E_{\|} / m-w_{s} \vec{v}\right) \phi f_{m}$ on $\Sigma_{\text {out }}$ is the mora exact posing of the problem. The less exact posing takes $\nu_{s} \rightarrow 0$. In either event, the response function satisties $\mathrm{E}_{\mathrm{g}}(16), \mathrm{D}_{t} \psi=Q_{\psi^{\prime}}$ with $Q_{\psi}$ assuring orthogonality, with initial condition

$$
\psi(0)=0 \quad \text {, }
$$


and boundary conditions

$$
\begin{aligned}
& \vec{s}_{B}=E_{m}\left(\hat{i}_{H} e E_{B} / m-v_{g} \vec{v}\right) \text {, on } \Sigma_{\text {out }} \\
& \vec{s}_{B}=0, \text { on } \Sigma_{\text {in }} \quad \text {. }
\end{aligned}
$$

Solving Bqs. (24) and (25) for $\psi$, and using $\#$. (23), we get (neglecting nonrf-induced contributions),

$$
\mathrm{N}_{R}=\int_{v} \mathrm{~d}^{3} \mathrm{v} \int_{0}^{=} a \tau \vec{\Gamma}(\vec{v}, t-\tau) \cdot \partial \psi(\vec{v}, \tau) / \partial \vec{v} .
$$

Note that $\overrightarrow{\psi(\vec{v}, t)}$ may be interpreted here as the probability with which a particle appears on $\Sigma_{\text {out }}$ by time $t$ given initial coordinate $\vec{v}$. For finite temperatures, eventually all particles would run away, since even those that slow down into the bulk distribution will ultimately scatter out of the bulk and run away. It is convenient, however, to label such electrons as stopped electrons, since they would run away on a much longer time scale. Suppase that the collision frequency were made artificially large as $v+0$ (or particles appearing near $v \neq 0$ were frozen and not allowed to run away). Then particles running away without passing through the bulk would be termed runaways, and the others would be termed stopped. The probability of a particle running away given initial coordinate $\vec{v}$ may then be expressed as

$$
R(\vec{v})=\Phi(\vec{v}, t \rightarrow \infty)
$$

This runaway rate has been obtained previougly through an equivalent formulation of the problem using Langevin equations. ${ }^{4}$ The numerical 
evaluation of the runaway rate was carried out by a Mante Carlo methor. Solving Eqs. (24) and (25) difectly, however, would be nuch easier.

It is often helpful to digtinguish the contributions of distinct groups of electrons to a given effect. For example, both runaway and stopped electrons contribute to the current. By identifying the separate contributions, one can determine if there is anything to be gained by increasing or decreasing somehow the number of runaways, possibly by controlling their confinement time. In Ref. 15, distingusishing these contributions facilitated a comparison betwen theory and experiment.

In order to deduce the contribution to an effect, say the current, due solely to stopped electrons, we make use of the function $R(\vec{v})$ defined in $F$. (27). We write the parallel current as $J=J_{R}+J_{S}$, where $J_{R}$ is the runaway current and $J_{S}$ is the current due to stopped electrons. We can write $J_{S}$ as

$$
\vec{J}_{s}=\int_{v} d^{3} v E_{m}(\vec{v}, t)\left\{v_{\|}[1-R(\vec{v})]+\varepsilon_{1}+\varepsilon_{c_{2}}\right\},
$$

where $c_{1}$ and $c_{2}$ are constants to be determined. Evidently, the appropriate Green's function, $\Psi_{s}$, for $J_{s}$ solves $E$. (16) with homogeneous boundary conditions and initial conation

$$
\Phi_{s}(\vec{v}, t=0)=v_{h}[1-R(\vec{v})]+c_{1}+\varepsilon_{c_{2}},
$$

with $c_{1}$ and $c_{2}$ determined by the orthogonality properties of $\psi_{g}$. using घq. (21), we then have $\left.J_{S}=-\left[\psi_{S},(\partial / \vec{\partial}\}\right)+\vec{\Gamma}\right]$.

The last exanple in this section illustrates the ease with which analytic solutions can sometimes be found for the response functions. Analytic expregsions are often available when the colligion operator can be written in 
the large-velocity 1imit. For example, the incremental conductivity due to the heating of superthermal electrons by $r f$ waves can be calculated analytically. This is the so-called "hot conductivity." The aimplifiration of the collision operator in the large-velocity limit is valid, because only the superthermal electron contribution to the current is important. In contrast, the Spitzer-Härm conductivity is not available analytically. Both Jlow and fast electrong contribute importantly to the Spitzer-Härm curcent and a drastic gimplifiction of the collision operator is not posgible.

In the high-velocity limit, the collision operator simplifies to

$$
C(\phi)=-\frac{\nu_{D} f_{m}}{2 u^{3}}\left[-u \frac{\partial}{\partial u} \phi+\left(\frac{1+\frac{\partial}{2}}{2}\right) \frac{\partial}{\partial \mu}\left(1-\mu^{2}\right) \frac{\partial}{\partial \mu} \phi\right],
$$

where $v_{0}=\omega_{p}^{4} \ln \Lambda / 2 \pi n v_{T}^{3}$ is a collision frequency, $u \equiv v / v_{T^{\prime}} \mu \equiv v_{\|} / v_{,} v_{T} \equiv$ $(\mathrm{T} / \mathrm{m})^{1 / 2}$, and $z$ is the ion charge state. In order to find the hot conductivity, we need to solve $\mathrm{Aq}$ (16) in the 1 imit $\vec{F}=\hat{i}_{\|} E_{\|} / m \rightarrow 0$. Also, since we wish to find the steady-state condretivity, rather than the transient effects, it is necegsary only to calculate the time integral of $\phi$ rather than $\psi$ itself.

Accordingly, define

$$
x(\vec{v})=\int_{0}^{\infty} \text { it } \psi(\vec{v}, \tau)
$$

where $\psi(\vec{v}, \tau)$ solves Bq. (16) with homogeneous boundary condi-ions and initial condition $\psi(\vec{v}, \tau=0)=$ ev $_{\mathrm{T}} \mathrm{u}$. Integrate Eq. $(16)$ from $\tau=0$ to $\tau=0$ to obtain

$$
-f_{m}\left(\vec{F} \cdot \frac{\partial}{\partial \vec{v}}\right) \chi-c(x)=f_{m} e v_{T} u \mu .
$$


where we noted that $\psi(\vec{v}, \tau) \rightarrow 0$ as $\tau \rightarrow 0$ (for finite conductivity), and that the constants $q_{1}$ and $q_{2}$ in $\mathrm{Eq}$. (16) are, in this case, zero. Expanding $x$ in a power series in $E_{H}$, i.e., $\chi=x_{0}+E_{H} x_{1}+\ldots$, allows us to so:ve Eq. (32) analytically. The first term gives the ri-driven current in the absence of a dc electric field. The second term gives the current that is bilinear in both the dc electric field and the rf power absorbed. The wave induced current is then given by

$$
J=\int_{v} d^{2} v \vec{r} \cdot \frac{a}{\partial v} x
$$

From Fa. (33), we can find the hot conductivity, $\sigma_{H^{\prime}}$ which is inentical to that given in Ref, 3.

\section{MODIFIED CHAPMAN-ENSKOG EXPANSION}

Although it cannot describe all the phenomena important to wave-driven plasmas, the expansion of Chapman and Enskog remains a simple and effective apptoach to describing transport impottant to the bulk of the electrons. The question is how we can modify this expansion so as to incorporate effects associated with the fast, collisionless electrons, especially those effects that can be described by Eq. (18) and its associated formalism. We seek an expansion that would reduce to the chapman-Enskog expansion in the 1 imit $\vec{P}+$ 0 , and would, for finite $\dot{\Gamma}$, describe adequately the dynamics of the fast electrons, In this expansion, the heating of the bulk electrons by the fast electrons, as well as other effects that link the two distributions, should play a role in the evolution of the Chapman-Enskog variables $(n, T, \vec{c})$ for $\vec{\Gamma} \neq$ o. (Here we use $\vec{c}$ to describe the drift of a Maxwellian distribution, with the understanding that all these variables, $n, T, \vec{c}$, must be specified 
separately for each species.l In this gection, we degcribe an expangion that meets these criteria. We remark, however, that this expansion, like the Chapman-Enskog expansion itself, is not a unique choice, altnough it does possess some desirable features.

we begin with Eqg. (1) and $\{2\}$, and we expand $f$ using the formall expangion parameters $\theta$ and $\delta$ (assumed small but eventually set equal to one), i.e., we expand

$$
f=f_{0}+\sum \theta^{j} f_{j}+\sum \delta^{k} k_{k}, \quad j, k \geqslant 1
$$

where the $f_{j}$ represent corrections primarily to the bulk electron distribution and the $h_{k}$ represent the wave-driven corrections. Accordingly, the collisions among the $f_{j}$ are assumed to take place on a much shorter time scale than collisions either between the $t_{j}$ and the $h_{k}$, or among the $h_{k}$. We shall order

$$
\begin{aligned}
& c\left(f_{j}, f_{k}\right) \sim \frac{1}{\theta}, \\
& c\left(f_{j}, h_{k}\right) \sim 1, \quad j>0, k>1 \\
& c\left(h_{j}, h_{k}\right) \sim 1, \quad j, k>1 .
\end{aligned}
$$

The $1 / \theta$ Ea. (35a) ordering is inherited from the Chapman-Enskog (C-E) approach, whereas the other ordering, we shall see, allows us to describe better the dynamicg of fast electrons.

In the C-E approach, the use of multiple time scales serves as a means of convenient bookkeeping. Here, in addition to the C-E time scales, we introduce a parallel scaling in $\delta$, i.e., 
23

$$
\frac{\partial}{\partial t}=\frac{\partial}{\partial \tau_{0}}+\theta \frac{\partial}{\partial \tau_{1}}+\theta^{2} \frac{\partial}{\partial \tau_{2}}+\ldots+\delta \frac{\partial}{\partial t_{1}}+\delta^{2} \frac{\partial}{\partial t_{2}}+\ldots .
$$

which facilitates the formal expansion. We assume the functional dependencies

$$
\begin{aligned}
& f_{0}=f_{0}\left(\tau_{0}, \tau_{1}, \tau_{2}, \ldots, t_{1}, t_{2}, \ldots, \vec{v}, \vec{r}\right) \\
& f_{j}=f_{j}\left(\tau_{0}, \tau_{1}, \tau_{2}, \ldots, \vec{v}, \vec{r}\right), j>1 \\
& h_{j}=h_{j}\left(\tau_{0}, t_{1}, t_{2}, \ldots, \vec{v}, \vec{r}\right), \quad j>1,
\end{aligned}
$$

Consider terms in the expansion of order $\theta^{k}$, which represent the ChapmanFnskog terms. To order $1 / \theta$, we have

$$
c\left(f_{0}, f_{0}\right)=0
$$

which has the drifting Maxwellian solution

$$
f_{\mathrm{o}}=\mathrm{f}_{\mathrm{m}}(\mathrm{n}, \mathrm{T}, \overrightarrow{\mathrm{c}})
$$

with $n, T$, and $\overrightarrow{\mathbf{c}}$ functions of time and space. To higher order in $\theta$, we have

$$
\begin{aligned}
& \theta^{\circ}: \quad c\left(f_{1}\right)=\frac{d}{d \tau_{0}} f_{0} \\
& \theta^{1}: \quad c\left(f_{2}\right)=\frac{d}{d \tau_{0}} f_{1}+\frac{\partial}{\partial \tau_{1}} f_{0}-c\left(f_{1}, f_{1}\right)
\end{aligned}
$$

where 


$$
\frac{d}{d \tau_{0}} \equiv \frac{\partial}{\partial \tau_{0}}+\vec{v} \cdot \frac{\partial}{\partial \tau}+\vec{F} \cdot \frac{\partial}{\partial \vec{v}} \cdot
$$

Fututions (40) represent the firgt terms of the Chapman-Engkog expansion. The $f_{j}, j \geq 1$ are chosen to contain no particles, current, or energy. Compatibility i:3 assured, order by order, by using

$$
\frac{\partial f_{a}}{\partial \tau_{j}}=\frac{\partial n}{\partial \tau_{j}} \frac{\partial f_{m}}{\partial n}+\frac{\partial T}{\partial \tau} \frac{\partial f_{j}}{\partial T}+\frac{\partial c_{c}}{\partial \tau_{j}} \cdot \frac{\partial f_{m}}{\partial t}
$$

and solving for $\partial_{n} / \partial \tau_{j}, \partial T / \partial \tau_{j}, \partial \tau_{j} / \partial_{j}$ to meet the requirements on the $f_{j}$. The $C-E$ quantities $n, T, \vec{t}$ then evolve according to, e.9.,

$$
\frac{d n}{d t}=\sum_{j=0} \theta_{j} \frac{\partial n}{\partial \tau_{j}}+\sum_{j=1} \delta^{j} \frac{\partial n}{\partial t_{j}}
$$

In the absence of contributions arising from $\partial / \partial t_{j}$, the terms $f_{0}, f_{1}$, $f_{2}$ are identical to the first three terms of the classic C-E expansion.

Matching terms of order $\delta^{j}$ allows us to describe additionally the effects of the wave-driven fluxes. For example, the first two equations in the expansion are

$$
\begin{aligned}
& \operatorname{th}_{1} \equiv \frac{d}{d \tau} h_{0}-c\left(h_{1}\right)=-\frac{\partial}{\partial t_{1}} f_{0}-\frac{\partial}{\partial \vec{v}} \cdot \stackrel{+}{\Gamma} \\
& I_{n}=-\frac{\partial}{\partial t_{1}} h_{1}-\frac{\partial}{\partial t_{2}} f_{0}+c\left(h_{1}, h_{1}\right) .
\end{aligned}
$$

Note that Eq. (44a) is identical to Bq. (8) for $f_{m} \phi$ except tiat $f_{0}$ is slightly more general here, that $h_{1}$ can be spatially dependent, and that the inhomogeneous driving term associated with the spitzer-Härm current has been accounted for in the $C-E$ part of the expansion. The last difference means 
thrit, consistent with our assunption of the low mean-free-path limit, : ignore any runaway electrons except those created by the waves. Those electrong could have been described had we ordered the term $\vec{F} \cdot \partial f_{0} / \partial \vec{v} \sim \delta$, however, that would take us further than wish away from the C-E approach. The differences between Fq. (44a) and Bq. (8) do not affect the transferability of the technique to solve these equationg. Boundary and initial conditions are imposed gimitarly. The adjoint method uged to golve E4. (B) may be employed both in Eqs. (44a) and (44b) and higher order equations in the K-expansion. The effect of the wave-driven fluxes on the bulk transport properties is then accounted for through the evolution equations for $n, T$, and $\vec{c}$, ag in Eq. (43).

While the above procedure accurateiy treats to firgt order both the waveariven fluxes am the bulk transport within the spirit of chapman and Fnskog, a question arises as to higher order terms in the expansion that are hybrid, i.e., bilinear in $h_{j}$ and $f_{k}$. There is a certain amount of arbitrarinegs in treating these hybrid terms; their main effect would be to correct somewhat the properties of the bulk plasma, rather than to change the dynamics of the fast electrons. Accordingly, it is most expedient to account for these terms by allowing then to drive the $f_{K^{*}}$ To insorporate them, we assume $\delta \sim \theta$. So, for example, the lowest order hybrid terms, whicl are bilirear in $f_{1}$ and $h_{1}$ [which are computable from Eqs. (40a) and (40b)], drive the third order C-E term $\mathrm{E}_{\boldsymbol{Z}^{\prime}}$ i.e.,

$$
\begin{aligned}
& c\left(f_{3}\right)=\frac{d}{d \tau_{0}} f_{2}+\frac{\partial}{\partial \tau_{1}} f_{1}+\frac{\partial}{\partial \tau_{2}} f_{0}-c\left(f_{2}, f_{1}\right)-c\left(f_{1}, E_{2}\right) \\
& -c\left(E_{1}, h_{1}\right)-c\left(h_{1}, E_{1}\right) .
\end{aligned}
$$


In such a manner, we may solve order by order both for $f_{j}$ and $h_{j}$, evolving $n, T, \dot{c}[e . g$, , through $E q .(43)]$ as we do so. Finally, we get the formal expansion parameters $\theta, \delta=1$. In practice, however, one may contemplate that only the firgt few terms of such an expansion are important.

IV. RESPONSE EUNCTIONS AND INFERENCE

In this section we adaress how the use of the response functiong developed here may help us in understanding plasma experimentg. There is an immediate and straightforward method of modeling the plasma. A second method uses the formalism developed here to develop important inferences.

The straightfor ward method is the traditional "transport code." Our best quesses as to transport coefficients, and other parameters thought to govern an evolving plasma, are collected to form a numerical description of the plasma. These best guesses could be arrived at either by theoretical inaight into fundamental plasma processes, or by empirical evidence gathered in many experiments, or by a combination of the two approaches. The development in Sec. $v$ indicates how the response functions nere may easily be incorporater in such a scheme. The traditional transport description that relates to tre bulk plagma is left untouched; however, effects associated with the rf-heating of fagt electrons may be treated systematically.

Let us illustrate this procedure by means of an example: Suppose that $r f$ waves are introduced into a tokamak plasma by means of waveguides or other injection devices situated on the periphery of the tokamak. The ray trajectories of the waves may be tracked numerically. The waves are followed until they are absotbed, giving up their enetgy and romentum to the absorbing plasma species at some point in the velocity space of that species and at some point in configuration space. The adjoint equations can be used to determine 
the amount of bulk heating and current generation per wave power absorbed. To determine the amount of wave power absorbed, a number of approximations may be used. Antonsen and frui ${ }^{19}$ assume that the damping is linear. A more precise approach is to use the one-dimensional quasilinear equations, which take into account the flattening of the velocity distribution in response to the waves. Although neither of these approsches is exact in its determination of the power absorbed, the ratio of current generated to power absorbed, which is often more imp:rtant, is an exact quantity.

Once the trajectory and the effects of the waves are determined, the bulk plasma properties may be evolved through transport equations. The result is a simulation of a plasma heating experiment. \{gee, e.g., Ref. 20$\}$.

In practice, simulating a plasma in this manner has not been particularly successful. The problem is that inherent in this approach is the multiplication of uncertainties. It is not absolutely certain what the wave phasing is at the plasma periphery; it is not certain whether these waves will be scattered by edge turbulence as they enter the tokamak; it is not certain that the plasma absorbs this radiation according to the approximate theory used; and it is not certain that other quant:ties governing the plasma heat, particle transport, or the background magnetic configuration are correcty given. The probability that cie simulation will correctly preaict an important experiment observable, however, is related to the product of the likelihoods that each process is correctly modeled. Nonetheless, the plasma transport code has undergone major development as a numerical tool' for designing and interpreting experiments.

The second method of employing the response functions derived here to explain plasma experiments is through the identification of inferences. Instead of attempting a complete numerical description of the evolving plagra, this method seeks to isolate and understand specific mechanisms., 
Consider, for example, the cage of the rt-heated plasma. Suppose that in the interior of the plasmas a wave-induced flux $t$ of electrons is produced in the resonant region of electron velocity space. The plasma fesponds in several ways. There may be, for example, an incremental production of current, runaway electrons, and plasma radiation at various angles and frequencies. Each of these quantities may be expressed as a function of $\vec{\Gamma}$ by use of the appropriate regponse function.

An understanding of the plasma can now be developed on the basis of conditional probabilities. Given that the wave power is absorbed by a certain group of electrong, a set of experimental observables may be recorded and related to each other. For example, an observed increase in the current density or poloidal field energy should be directly correlated with an increase in rariation at certain frequencies. The precise mechanism of wave propaqation and damping need not be uncovered, then, in order to infer the location and velocity of the resonant electrons, as well as other local plasma parameters governing the radiation.

similarly, if it is the theory of the radiation that is to be checked, then instead of plotting the observed radiation vs input power, one might plot, e.g., the obgerved radiation vs. current-drive efficiency, or one might plot the observed radiation at one angle and frequency vs. that observed at a second angle and frequency. In these examples, one achieves an improved unciergtanding of specific plasma processes, without the necessity of a complete picture of the evolving plasma.

The response functions derived here are especially suited for such an approach. As a numerical tool, the identification of such inferences and correlations can be of great help in understanding experinents. After all, such a tool mimics most clogely the likely thought processes of the plasma 
experimenter. Faced with an unusual experinental result, the experimenter is far more likely to attempt crude pattern matching and other correlations to reduce data, than to simulate conceptually the complete picture of the plasma evolution. A numerical tool that aids the experinenter in his natural approach to explaining an experiment may be of more direct use than the far mote ambitious goal of providing a coaplete numerical simulation.

The above approach lends to meagurements of plasma radiation far more importance than might otherwise be suspected. These measurements are of little use ir the construction of a numerical simulation. On the other hand, they could provide a wealth of infotmation about the absorption of waves in $P$ Ariven plasma.

\section{SUMMARY AND CONCLUSIONS}

This paper concerns itself with the description of effects associated with fast, relatively collisionless electrons, whose mean-free path may be long compared to other lengths of interest. It is possible that these few, fast electrons will contribute importantly to plasma transport, particularly in the case of an intensely rf-driven plasma. Intense rf waves interacting primarily with fast electrons produce particle fluxes in velocity space that can substantially alter the distribution of electrons. The result may he a large change in the plasma current, aynchrotron radiation, bremsstrahlung, runaway production, or other quantities associated with the transpori of fast electrons.

In order to calculate the plasma response to wave-induced fluxes in velocity space, we linearized the inhomogeneous dynamic Boltzmann equation. Although the linearized equation is not much more easily solved numerically. than is the original equation, it is possible, because of the linearization, to find the Green's functions for quantities of interest. In sec. III we 
identified an adjoint operator, defined over a suitable inner product, that allowed us to generate adjoint equations for calculating these quantities. By imposing initial and boundary conditions on the adjoint equation, plasma responses may be calculated, via Br. (23), for arbitrary wave-induced fluxes $\vec{\Gamma}$.

An important simplification in the practical application of Eq. (23) arises from our ability to infer information about $\vec{I}$ from the resonance condition, as discussed in sec. II. This allows us to compare, for example, likely plasma responses per power dissipater as the phasing of the waves is varied.

We gave several examples in the use of these response functions in sec. IV. These examples showed how useful adjoint equations may be defined, an: how, in certain limits, analytic solutions are available.

The responses assogiated with these fast electrons are not fluid effects, and are not naturally found in small mean-free-path expansions of the Boltzmann equation, such as the expansion of Chapman and Enskog. In Sec. v, however, we showed how the $C-\Sigma$ expansion may be modified to account for the wave-driven Eluxes. An expansion, formally in two small parameters, was proposed: the frequently colliding bulk particles are treated fluidlike, exactly as in the C-E expansion, while the fast, long-mean-free-path electrons evolve subject to external forces and subject to collisions predominantly with bulk electrons and ions. The description of the fast electrons is faniltated by the use of adjoint equations, and the effect of these electrons on the hulk transport processes appears in the higher order terms descrining the bulk particles. 
Although the responge functions derived here can be directly implemented in existing transport codeg that simulate completely the evolving plasma, we speculate that i more advantageoug use of this work is through the development of a set of correlationg from which plagma behavior might be inferred.

\section{ACKNONLEDONENTS}

It is a pleasure to acknowledge stimulating and helpful discussions with C. F. F. Karney, This work was supported by U.S. DoE Contract \#DE-ACO2-76CHO3073. 
REFERENCES

S. Chapman and $T$. Cowling, The Mathematical Theory of Non-uniform Gaseg, $3^{\text {td }}$ edition (Cambridge university Press, Cambridge, 19701.

2 L. Spitzer, Jr, and R. Härm, Phys, Rev, 59, 977 (1953).

3 N. J. Fisch, Phys. Fluids 28, 245 (1985).

4 T. Ohkawa, Nucl. Eusion 10, 185 (1970).

5 D. J. H. 4ort, Plasma phys. 13, 258 (1971).

6 w. J. Fisch, Phys, Rev, Lett. 41,873 (1979),

7 N. J. Fisch and A, H. Boozer, Phys. Rev. Lett. 45,820 (1980).

g T. M. Antonsen Jr, and K. R. Chu, Phys. Fluids 25, 1295 (1982).

9 S. P. Hirshman, Phys. Fluids 23, 1238 (1980).

90 M. Taguchi, J. Phys. Soc. Japan 51, 1975 (1982).

11 M. Taquchi, J. Phys. Soc. Japan 52, 2035 (1983).

12 T. M. Antonsen Jx, and K. Yoshioka, Bul1. Am. Phys, Soc, 29 (8), 1323 (1984).

13 F. C. Jobes et al., Princeton Plasma Physics Laboratory Report PPgL-2200 (Eebruary, 1985).

14 N. J. Fisch and C. F, F. Karney, Fhys. Rev, Iett (to be published).

15 C. F. F. Karney, N. J. Fisch, and F. C. Jobes, Princeton Plasma Physics Labori jory Report PPPL-2952 (October, 1984).

H. Grad, Phys. Fluids 6 , $147(1963)$.

17 B. B. Robinson and I. B. Bernstein, Ann. Phys. (Ny) 18, 110 (1962).

t8 M. N. Rosenbluth, R., D. Hazeltine, and F. L. Hinton, Phys. Fluids 15,116 $(1972)$.

19 T M. Antonsen, Jr, and B. Fui, IEEE Trans. Plasma Sci., Ps-12, 118 (1984).

20 P. T. Bonoli, Massachusetts Institute of Technology Report PFC/RR $-84-5$ $(1904)$. 


\section{REPAODUCED FROM \\ BEST AVAILABLE COPY}

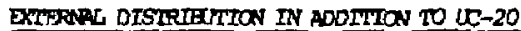

Plasia Res Lab, Austra Nat'l Univ, AusIRALIA Dr. Erark J. Paolont, Dniv of rollongong, AUSIRAIIA Prof. I. $R$. Jones, Flinders briv., ALSTRALIA prof. M.H, gcennan, Uhiv sydney, australma Prof. E, Cap, Inst Theo Fhys, Austrih ProE. Erark Verheegt, Ingt theoretische, EEIJIIM

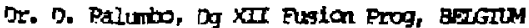
Fole Royale Milttairn, Iab de Phys Plasmas, BryGIM Dr. F.t. Sakanaka, Utiv Fstahial, EFrrII. Dr. C.R. James, thiv of Alberta, Curror prof. J. Teidtmann, Univ of Hontreal, Caspa Dr. म.M. Skarggard, thiv of Sagkatchewar, CAnpas Prof. S.R. Smenivasan, University of Calgary, CANADA Prof. Tuior \%. Johnston, IRRs-Eneryie, CANPos Dr. Hares Barnand, Univ Britlsh Colunbia, CARDA Dr. M.P. Bachynski, MPB Technologies, Inc., GARDA Chalk River, Nucl Iab, CANADA

Thengur Ii, $\mathbf{W}$ Inst Ptysics, CHDA

Library, Tsing Hua University, CHIlla

tibrarian, Instinute of Fhysics, CHDA

Inst Plasin Fhys, Acadenia Sinica, OmA

ir. Peter Lukac, Konenskeho bniv, CRofostovakTA

The Librarian, Culhan Laboratory, aNGAND

Prof. Schatonan, osservatoire de NLCe, FraxcE

J. Radet, CEN-BPG, FRANCE

44 Dupas Library, A4 Dupas Libracy, FRance

Or. 'Ior Mul, Acadenty Albllographic, Hax, kaNG

Preprint Library, Cent Res Inst Phy, Huvary

Dr. S.K. Tretan, Panjob University, BDTA

Dr. Indra tohan Lal Das, Banaras Hirka Univ, INDIA

Dr. I.K. Chavda, south Gujarat Untv, DNIA

DE. ILK Ghajlani, vikoran thiv. IIDTA

55. B. Dasgrupta, Saha Inst, IDDIA

Dr. P. Kas, Physical Regearch Lab, RDIA

DT. Phillip Rosenau, Istael Inst Tech, ISRAFI

Prof. S. Gypeman, Rel Aviv thiwersity, ISRAEI

Prof. G. Rostagni, Univ Di Padovm, TIMLX

tibrarien, Int'l Ctr theo gtye, IMLY

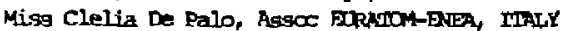

Biblioteca, del OR EPATOA, ITLY

DC. H. Yamato, Joghiba Fes \& Dev, JMPAN

Direc Dept Lg. Tokanak Dev. JAERI, JAPAN

Prof. Notisyuli Inove, University of Tokyo, JAFAN

Pesearch Info Center, Nagoja thiversity, JAPAN

Prof. Kyoji Nishikaw, Univ of Hicoshdma, JAPAN

Prof. Sigeru Hori, JaterI, DPAN

Tibrary, Kyoto University, JAPAN

Pmof. Ithito Kawkani, Nihon Univ, IAPAN

PruE. Satoohi Itoh, Kyoh thiversity, JaFAl

Or. D.I. Chol, Actv. Ingt Sci \& Tecti, KOREA

Teot Info Diviston, KAERI, KOREA

jiblitothesk, Fom-Inst voor Plasm, NEIMLRLANDS

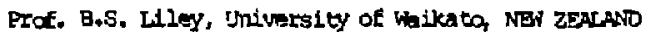
Prof. J.A.C. Cabral, Inst Syerio Tecn, Fortucht De. Octavian Petrus, ALI ORA University, POAANIA. Pnuf. MA. Hellberg, thimersity of Natal, $D$ AFRICA Dr. Johan de villiers, Dlaena Phygica, Hucor, so AfRTCA Fuian Div. Tilbrary, JEN, SPAnN Prof. Fans wilhelmeon, Chalmers Univ Tach, SWEDNN

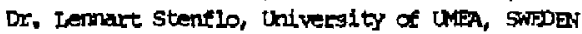
Ifbrary, Royal Inst tech, ShDoDN

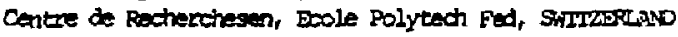
Dr. V.T. Toldk, Wharkou Phys Tech Ins, ISSR

Dr. D.D. Ryutor, Siberian Acad Sci, USSR

Dr. G.A. Eliseov, Kirchatov Ingtitute, I5SK

Dr. V.A. Fivinitich, Inst Electro-Phygical, USSR Insti urte Gen Physicy, USSR

Prof. T.J.M Boyd, Univ college N wales, WALES Dr. K Schindler, Puhr Inivergitat,., GEPMANY Mrelear Res Fatab, Julich Ltd, H. GePMANY

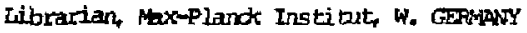
Albliothek, Inst Plasmaforschung, w. GEPANY Prof. R.K. Janev, Ingt Phys, noustnita 